\author{
Lars Johnsen*
}

\title{
The hypertext-based grammar \\ - or the grammar of tomorrow
}

\section{Background}

Over the past couple of years we have witnessed an enormous increase in the availability of electronically stored information resources relevant to the teaching of foreign languages at all levels in the educational system: electronic dictionaries, terminological databases, interactive language learning programmes, machine readable corpora, online services, etc.

Many of these information resources are hypertext-based. Hypertext may be described as a technology or medium for organizing and presenting electronically stored information elements or documents in a network of information nodes. There are several reasons why hypertext is increasingly used in educational contexts. One of the main reasons is flexibility. Firstly, hypertext means that authors are relatively unconstrained by structural form. Besides employing traditional forms such as sequence, hierarchy and grid, authors are free to link information items in an associative fashion (see Horton (1994)). Secondly, hypertext may provide the reader with a number of ways of accessing information more effectively. Query functions facilitate the retrieval of specific information items while various navigation and orientation tools make it possible for the reader to traverse (parts of) the hypertext network in a non-linear manner. Thirdly, hypertext offers alternative ways of conveying information. Compare, for instance, Whalley (1993:12) who says:

The most significant pedagogic feature of hypertext is its malleability:

it may change over time and it is capable of offering multiple perspectives on a particular domain. It is possible to use hypertext to

* Lars Johnsen

Handelshфjskole Syd

Institut for Erhvervssprog og Sproglig Informatik

Engstien 1

DK-6000 Kolding 
present, and then represent, ideas in ways that are difficult to achieve in print."

Perhaps the most interesting example of hypertext today is the World Wide Web, a rapidly growing global network of information resources accessible on the Internet.

\section{Aims}

The present article discusses the potential of hypertext in relation to one of the classical tools in language learning environments, namely the grammar book. The aim of the article is two-fold. One aim is to demonstrate how the functionality of hypertext might be used to support, and even optimize, certain activities and tasks associated with the acquisition of grammatical content. Another aim is to sketch how the technology of hypertext may contribute to more efficient, effective and innovative management of language resources in educational settings.

\section{Access to information}

Grammars are, among other things, works of reference. The point is made quite neatly by Jensen (1990:13) in the preface to his book on Spanish grammar entitled Spansk basisgrammatik:

"Only grammarians would ever dream of reading a grammar like a novel, from cover to cover. To everyone else a grammar is a handbook, a work of reference in which one can quickly find the information one needs."

(my translation)

How quickly and effectively an end user may retrieve information in a traditional grammar is contingent on the quality of the access structure of the document. The access structure consists of a number of text elements which, to varying degrees, determine the accessibility of the individual information items:

* the macro-structure of the text (the way information units are organized)

* the micro-structure of the text (the internal structure of information units)

* information design (typography, layout and the use of visuals such as tables, lists, tree structures, etc.) 
* headings (position, font type and size, verbal content)

* meta-information (table of contents, index, headers and footers)

In a hypertext-based grammar, more interactive information retrieval tools can be made available. These include query functions, navigation tools and orientation facilities.

A query function is a facility which makes it possible for the end user to search for certain key terms, examples or phrases - or combinations of these - in the grammar. Queries may apply to the entire document or to certain parts of the text and scopes may be set to limit the search even further. Thus, scopes can be defined in terms of the structural elements of the text or its content elements. For example:

i) find all occurrences of transitive or intransitive in subheadings in the chapter entitled Verbs

ii) find all occurrences of provided that in the examples given in the text

Navigation tools allow the reader to move non-linearly among information units in the text ('browsing'). The most typical example of a navigation tool in a hypertext is the link, a clickable text element connecting two or more information units. The activation of a link may either result in the reader actually 'hopping' from one place to another in the network or the display of one or more information units in a separate window. Links may be classified as intra-hypertextual, interhypertextual or extra-hypertextual depending on whether they link information segments in the same information unit, the same document or in different documents (Kuhlen (1991:107)).

Links can be used in various ways in a hypertext-based grammar. The most obvious idea is to implement cross references as links. With a click on a cross reference the user gains access to the section referred to by the cross reference. Links may also be used to create paths to supplementary information such as explanations, examples and translations. Grammatical terms might be linked to definitions, footnotes to information units and authentic examples might be shown in their full context.

Links are also useful if an author wishes to guide the reader through an information space consisting of non-sequentially ordered units. A guided tour makes it possible for the reader of a grammar of English to get an introduction to, say, important differences between American 
and British English even if these differences are not dealt with in the same chapters or sections. A guided tour may be implemented as a genuine tour, that is, as a sequence of jump links sending the reader from one relevant information unit to another in the text or it may be constructed as a virtual document. A virtual document is a group of electronically stored but physically dispersed information segments which may be extracted from the text and viewed and read as a whole.

One can also envisage the use of extra-hypertextual links in an electronic grammar. These may comprise connections to electronic dictionaries, terminological databanks, electronically stored textbooks or text corpora containing authentic examples. Such resources may be local (located on the student's own PC or a local area network) but not necessarily so. With the appropriate hardware and software, links may be established to relevant information resources on the World Wide Web such as databases or teaching materials. For instance, a teacher of English might like to include in his grammar a link to information units in Purdue University's "Online Writing Laboratory" (OWL), an information system providing guidelines on miscellaneous topics related to writing (punctuation, grammar, non-sexist language, formats for citing sources, etc.). Or he might like to set up a query link to a specific text database at some American law school to illustrate the use of the modal shall in legal discourse.

Although navigation tools provide an expedient way of accessing information, totally unguided browsing may lead to disorientation on the part of the reader, who may become lost in hyperspace. It is therefore important that the hypertext-based grammar is endowed with a number of (electronic) orientation tools helping the reader address questions such as:

* Where am I?

* What is this about?

* How do I find ...?

* How do I get back to ...?

* Where have I been so far?

Orientation tools may serve three purposes. One is to give the reader a general idea of the contents of the grammar or parts of the grammar. Another is to indicate where specific items are located in the online document. Last but not least, orientation facilities may state the reader's 
current position in the network or display a list of information units already frequented by the reader. Electronic orientation facilities include:

* expandable/collapsible table of contents

* electronic bookmark

* history (list of information units already seen by the user)

* trail (list of links or queries previously activated by the reader)

* graphical browser ((clickable) map depicting the visual structure of the grammar).

\section{Communication and interaction}

Naturally, access is not everything. The usefulness of a grammar as a learning tool is primarily determined by the validity of its contents and the way these contents are communicated. As mentioned above, the electronic medium is a more flexible vehicle for conveying information than paper. Unlike a traditional printed text in which all of the contents - no more no less - are presented to the reader, hypertext may contain several layers of information. These layers may be unfolded by the reader or they may be kept hidden depending on whether the reader wants to explore the details of the material or just wishes to get the gist of the text.

As far as hypertext-based grammars are concerned, it would perhaps be a good idea to let the reader himself decide how many examples, references and exceptions to general rules he wants to see. It would no doubt also enhance the usability of (hypertext-based) grammars if the markedly verbal form of communication was supplemented with more visually oriented discourse elements such as tree structures, lists, diagrams and tables. These might be placed in pop-up windows and activated if needed.

Another advantage of computer-based learning materials is the possibility of interaction between reader and text. Interaction in an electronic grammar may take place if the student is given the opportunity to work with exercises and tests with built-in feedback. The chief function of such tests is to provide the student with an assessment of how well he has understood a certain topic dealt with in the grammar, to point out what his specific problems are (diagnostic tests) and perhaps what course of action might be appropriate in the circum- 
stances (what information units to read, what level of detail to select, etc.). It should be mentioned, however, that only comparatively closed types of tests (true/false questions, multiple choice exercises, selection sets, etc.) may be evaluated satisfactorily online. The evaluation of more open-ended assignments is still a matter for the teacher.

\section{The grammar as construction kit}

Although the functionality of a hypertext-based grammar may be said to be greater than that of a traditional grammar in a number of ways, the two types of text have essentially the same function in the learning environment. They are both used as an information bank, which, according to Perkins (1991:18), is any resource in the learning environment which 'more than anything else, serves as a source of explicit information about topics'.

Nevertheless, one can also imagine the hypertext-based grammar being used more actively in the learning process. One possibility is to design the grammar as a kind of construction kit (see Perkins (1991:19)). That is to say, as a toolbox containing functions and elements which more directly support the student in constructing grammatical knowledge structures and in relating these knowledge structures to other types of linguistic knowledge. Thus, the student might be given access to a range of tools enabling him to mark up, compare, link, group and annotate individual information elements in the grammar as well as integrate these elements with information items found in external sources such as dictionaries, text books, texts for translation and so forth.

Working with grammatical resources in this way might either result in a number of more or less customized 'personal language databases' built up by students or a large, dynamic information base constructed by teachers and students in collaboration. Here the vision is an electronic network of language resources, including grammatical information, being constantly updated and expanded as teachers and students discover new thematic patterns in the material, come across interesting examples or find useful references. The grammar as groupware, so to speak.

The actual product, however, is not the most important thing. What is significant in my mind is that by promoting a (mildly) 'constructivist' 
approach in these matters, we may succeed in motivating students to take on a new role in their language learning activities, a role which will not only involve more activity on their part, but hopefully also generate a greater awareness of, and responsibility for, the learning process itself (see Cunningham et al. (1993)).

\section{Learning with hypertext-based grammars}

So far, I have attempted to describe some of the practical uses to which a hypertext-based grammar might be put and the kinds of learning tasks its functionality might support. A framework for thinking about the pedagogic aspects of computer-based learning materials in general, and hypertext in particular, is found in Hammond (1993). This framework is based on a three-dimensional model characterizing relevant properties of educational software. The three dimensions, control, engagement, and synthesis are defined in the following way:

"Control refers to the degree to which the learner rather than the
system controls exposure to learning materials, the particular learning
activity or strategy. Engagement refers to the extent the learner is
required to process the materials actively rather than passively. Syn-
thesis refers to the nature of the learning activity: does it require the
learner to create materials or relationships rather than merely observe
them? These last two dimensions are not independent since creative
tasks generally require active engagement, although the converse is
not true."

(Hammond (1993:64))

In terms of hypertext-based grammars, control can be said to be the degree of freedom the reader has when accessing grammatical information. At one end of the spectrum the reader is entitled to unbridled browsing and searching and at the other he is only allowed to follow carefully planned, sequentially ordered information structures. Engagement refers to the difference between reading 'passive text' on the one hand and interacting with it, for instance through the use of tests, on the other. Finally, synthesis may reflect the extent to which the grammar may be used as a construction kit.

To determine what degree of control, engagement and synthesis is to be incorporated in the functionality of a hypertext-based grammar under construction, an author must take a number of factors into consideration. For example: 
* Who is the intended audience (advanced learners, beginners)?

* What is the primary purpose of the grammar (work of reference, course text, cramming tool)?

* What learning activities does the author wish to support (perusal, skimming, interaction, collaboration)?

* In what educational context will the grammar be used (distance learning, self study, class room teaching)?

\section{Information management}

In addition to its role as a potentially powerful learning tool, hypertext may contribute to more efficient, effective and innovative management of language resources in educational settings. This is especially true if the grammars of tomorrow are designed and constructed as what we might call single source grammars. A single source grammar may be described as a multi-functional information base containing (multilingual) grammatical information modules which may be published in several media.

Modularity in a single source grammar means that individual modules are designed as a kind of reusable Legos. Individual modules may occur in different textual structures at the same time thus creating a number of virtual documents. For instance, a module describing how singular collective nouns in American English and in British English differ with respect to their compatibility with singular or plural verb forms, might occur simultanously in virtual documents with titles such as:

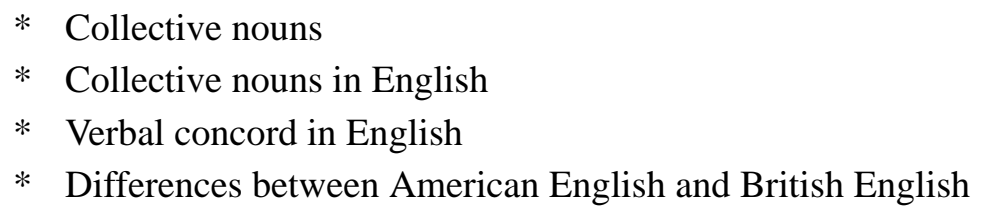

Also, reusability is conducive to the creation of new information products as information units are grouped in novel ways. Examples of new information products generated on the basis of a single source grammar might be minimalist, contrastive or multilingual grammars, glossaries containing definitions of important grammatical concepts or collections of authentic examples for specific purposes. 
An implication of single source grammars may also be more flexibility in terms of production, distribution and maintenance. As for production, a modular approach for instance means that authors can work in collaboration and that publication may take place in serial form. With respect to distribution, a single source grammar may be constructed in such a way as to allow the publication of paper-based materials as well as electronic documents from the same set of files. Furthermore, it can both be implemented as an inhouse information base placed on a local area network or as a resource which can be accessed by means of the Internet or World Wide Web. The latter solution has the advantage of catering for the information needs of ordinary students and distance learners alike. Finally, maintenance is likely, at least in theory, to become less burdensome since a module containing outdated or inaccurate information can just be plugged out and a new one put in without other modules having to undergo major revisions.

\section{Concluding remarks}

Now, what does it take to create usable hypertext-based grammars that may function as electronic reference documents, interactive language learning programmes, virtual documents, construction kits or single source grammars?

Firstly, needless to say, the appropriate development tools must be available. To choose the right product(s) is in itself no easy job since there is a plethora of hypertext systems, infobase management systems, text editors and programming environments, let alone third-party addons, on the market today each with its own functionality, interface and (in)compatibilities with other products and platforms. What is also important to realize is that no single product is likely to contain the range of tools needed to implement all the functions mentioned above.

Secondly, we undoubtedly need to adopt design and development methodologies which can ensure a more consistently modular information structure than is met with in traditional paper-based grammars (see Johnsen (1996)) as well as overcome the inherent limitations of the computer screen as a communication interface (the problems of a smaller reading area, the poorer resolution of text and the fact that the reader has to make sense of the text through a tunnel, as it were). 
In my opinion, one way forward is to explore the possibilities of adopting conceptual tools from the field of technical communication where experimental research in electronic text usability has led to the formulation of a number of guidelines and principles for online information design (see Horton (1994)). In particular, it would seem worthwhile to find out if, or to what extent, elements from modular writing methods such as Information Mapping (Horn (1989)) or Structured Documentation (Weiss (1991)) originally designed for business and technical communication might be adopted for the kind of language technology we have in mind here. What is interesting about a methodology like Information Mapping is that it not only provides a number of guidelines on how to structure and present information in well-defined units but also attempts to describe how certain information types - processes, procedures, concepts, etc. - may be identified and communicated in an effective manner. And what is perhaps particularly interesting in this connection is that Information Mapping specifically deals with possible ways of presenting information types that can be said to occur regularly in grammars, namely:

* structure ('The verb phrase consists of ...')

* fact ('Specific reference is used when the speaker refers to a specific member or manifestation of a category.')

* concept ('A declarative clause is ...')

* classification ('There are three main types of clause: ...').

Examples taken from Preisler (1992)

Lastly, in order to be processed electronically and accessed through global networks, information modules and information categories must be identified and coded in a so-called markup language. If the grammar is to be published as a document on the World Wide Web, it must be coded in, or converted to, HTML (HyperText Markup Language), a format compatible with the specifications of SGML (Standard Generalized Markup Language), a general markup language for describing and exchanging documents across disparate products and platforms.

\section{References}

Cunningham, D.J., Duffy, T.M. and Knuth, R. A. (1993): The Textbook of the Future. In McKnight, C., Dillon, A. and Richardson, J. Hypertext. A Psychological Perspective. Ellis Horwood Limited. 
Hammond, N. (1993): Learning with Hypertext: Problems, Principles and Prospects. In McKnight, C., Dillon, A. and Richardson, J. Hypertext. A Psychological Perspective. Ellis Horwood Limited.

Horn, R. E. (1989): Mapping Hypertext. The Lexington Institute.

Horton, W. (1994): Designing and Writing Online Documentation. Second edition. John Wiley \& Sons, Inc.

Jensen, K. (1990): Spansk basisgrammatik. Akademisk Forlag.

Johnsen, L. (1996): From text to hypertext: Developing foreign language grammars for technology supported LSP-learning environments. (Forthcoming).

Kuhlen, R. (1991): Hypertext. Ein nicht-lineares Medium zwischen Buch und Wissenbank. Springer-Verlag.

Perkins, D. N. (1991): Technology Meets Constructivism: Do They Make A Marriage. Educational Technology/May 1991.

Preisler, B. (1992): A Handbook of English Grammar on Functional Principles. Aarhus University Press.

Weiss, E.H. (1991): How to Write Usable User Documentation. The Oryx Press.

Whalley, P. (1993): An Alternative Rhetoric for Hypertext. In McKnight, C., Dillon, A. and Richardson, J. Hypertext. A Psychological Perspective. Ellis Horwood Limited. 